\title{
Creación de mapas acústicos en los cantones de Heredia, Alajuela y Belén, como indicadores de la contaminación sónica.
}

\section{Development of acoustic maps in Heredia, Alajuela and Belén counties, as indicators of noise pollution.}

\author{
Ligia Bermúdez-Hidalgol \\ José Castro-Solis ${ }^{2}$ \\ Karla Vetrani-Chavarría \\ Igor Zúñiga-Garita ${ }^{4}$ \\ Manfred Murrell-Blanco ${ }^{5}$ \\ Douglas Barraza ${ }^{6}$ \\ Manuel Méndez-Flores ${ }^{7}$ \\ Universidad Nacional, Costa Rica
}

\section{Resumen}

El presente artículo corresponde a la conformación de mapas acústicos basados en la toma de datos de ruido en los cascos urbanos de los cantones de Heredia, Alajuela y Belén en Costa Rica. Para esto se realizó un muestreo, utilizando sitios de denuncia por contaminación sónica en Alajuela y Belén,

1 Doctora en Ingeniería Industrial, Costa Rica, Escuela de Ciencias Ambientales, Universidad Nacional, Costa Rica. Correo electrónico: ligia.bermudez.hidalgo@una.cr

2 Máster en Conservación y Manejo en Vida Silvestre, Costa Rica, Escuela de Ciencias Ambientales, Universidad Nacional, Costa Rica. Correo electrónico: jose.castro.solis@una.cr

3 Máster en Administración de Proyectos, Costa Rica, Escuela de Ciencias Ambientales, Universidad Nacional, Costa Rica. Correo electrónico: karla.vetrani.chavarria@una.cr

4 Máster en Administración de Proyectos, Escuela de Ciencias Ambientales, Universidad Nacional, Costa Rica. Correo electrónico: igor.zuniga.garita@una.cr

5 Máster en Ingeniería en Recursos, Escuela de Ciencias Ambientales, Universidad Nacional, Costa Rica. Correo electrónico: manfred.murrell.blanco@una.cr

6 Máster en Salud Ocupacional con énfasis en Higiene Ambiental, Escuela de Ciencias Ambientales, Universidad Nacional, Costa Rica. Correo electrónico: douglas.barraza.ruiz@una.ac.cr

7 Máster en Ingeniería Industrial, Escuela de Ciencias Ambientales, Universidad Nacional, Costa Rica. Correo electrónico: manuel.mendez.flores@una.cr.

Este artículo corresponde a la ponencia presentada en el I Congreso Centroamericano de Ciencias de la Tierra y el Mar, realizado en San José, Costa Rica, del 13 al 16 de noviembre de 2017. 
mientras que en Heredia se utilizaron los vértices de cada uno de los cuadrantes que conforman el distrito Central como sitio de muestreo. El resultado más importante obtenido en este estudio está referido a la identificación en su mayoría de sitios con niveles de ruido que sobrepasan los límites permisibles establecidos en el Reglamento de control de ruido que se pueden observar gráficamente en los mapas acústicos. Esto lleva a la conclusión de que los mapas acústicos representan una herramienta fundamental para el control de ruido y planificación de estrategias de mitigación y confinamiento de áreas de impacto por contaminación sónica.

Palabras clave: Alajuela, Belén, contaminación acústica, Heredia, mapas acústicos.

\begin{abstract}
The present study corresponds to the conformation of acoustic maps based on the collection of noise data in the urban centers of Heredia, Alajuela and Belén, in Costa Rica donwtown. Therefore, a sampling was carried out using sites of complaint for noise pollution in Alajuela and Belén, while in Heredia the vertices of each quadrants in Central District were used as a sampling site. The most important result obtained in this study is related to the identification of mostly sites with noise levels that exceed the permissible limits established in the Regulation that can be observed graphically in the acoustic maps. This leads to the following conclusion the acoustic maps represent a fundamental tool for the noise control and to plan the mitigation strategies and confinement of noise pollution areas.
\end{abstract}

Keywords: Alajuela, Belén, Noise pollution, Heredia, acoustics maps.

\title{
Introducción
}

Los acelerados cambios en la economía global, los flujos migratorios de personas, y la ocupación de nuevos terrenos en pro del desarrollo social han modificado la estructura tradicional del paisaje, lo que a su vez ha implicado la exposición a nuevos niveles de ruido considerados como contaminación ambiental. La acción del ser humano ha cambiado considerablemente muchos ambientes alrededor del mundo produciendo un incremento del ruido como una de las principales consecuencias del desarrollo económico (Farina, 2014). La continua expansión de la contaminación acústica ha reducido drásticamente los espacios libres de ruido antropogénico y las oportunidades de disfrutar zonas tranquilas en medio de la naturaleza, al mismo tiempo atenta contra la salud de los ecosistemas (Iglesias, 2014; Lynch, Joyce y Fristrup, 2011).

El sonido es una forma de energía mecánica propagada en forma de ondas longitudinales que producen una sensación auditiva al causar cambios en la presión, por ejemplo, del aire (Giménez, 2007). Físicamente, no hay diferencia entre sonido y ruido, sin embargo, el ruido se define como 
Ligia Bermúdez-Hidalgo - José Castro-Solís - Karla Vetrani-Chavarría - Igor Zúñiga-Garita

Manfred Murrell-Blanco - Douglas Barraza - Manuel Méndez-Flores

Creación de mapas acústicos en los cantones de Heredia, Alajuela y Belén, como indicadores de la contaminación sónica.

un sonido voluntario o involuntario que al añadirse a una señal acústica disminuye su capacidad de ser percibida o interpretada adecuadamente.

La contaminación acústica, principalmente en los grandes centros urbanos del orbe, representa uno de los principales problemas de salud por el daño auditivo y efectos que genera sobre el cuerpo humano, convirtiéndose en un tema de salud pública (Kurakula 2007, MER 2011, Oliviera et al, Szczodrak et al 2013), concretamente referido a la pérdida auditiva de la gente que vive en ellos (Gray, 2017).

El propósito de la presente investigación está enfocado en la determinación de los niveles actuales de ruido en distintos puntos del casco urbano de los cantones de Heredia, Belén y Alajuela, Costa Rica, utilizando métodos no convencionales de medición. Esta información permitió la conformación de mapas acústicos, donde se determinaron varias categorías, una primera categoría que incluyen sonidos de gran intensidad que abarcan actividades como fábricas donde una exposición prolongada puede producir pérdida auditiva y trastornos fisiológicos, y una segunda categoría que es más imprecisa, ya que considera elementos como ruido vehicular, maquinaria, voces humanas, los cuales son variables dado que este depende de la actividad que se desarrolle.

\section{Marco teórico - conceptual}

El sonido se define como sensación percibida por el oído humano, producto de las rápidas fluctuaciones de la presión del aire que, por lo general, son producidas por objetos que vibran y transmiten esta vibración a las partículas del aire (Ochoa, 2009). A partir de este se establece el concepto de ruido que es definido como un sonido indeseado y consecuentemente molesto, que puede producir una afectación fisiológica y psicológica en uno o más receptores (Iglesias, 2014). El ruido como fenómeno físico es un movimiento ondulatorio producido en un medio elástico por una fuente de vibración que provoca pequeñas variaciones de la presión atmosférica en el oído (Bovea et al., 2011).

Considerando esto se define el nivel de presión sonora que es una medida logarítmica de la presión acústica efectiva de un sonido con respecto a un valor de referencia (Farina, 2014). La unidad de medida correspondiente es la de presión, es decir, el Pascal (Pa). El ser humano es capaz de percibir presiones que van desde los $20 \mu \mathrm{Pa}$ hasta los $100 \mathrm{~Pa}$, lo que establece una 
relación entre la máxima y la mínima presión sonora de 1000000 de veces. Por lo que resulta conveniente el uso de una escala logarítmica, cuya unidad es el decibelio $(\mathrm{dB})$, la cual permite evitar el manejo de número muy pequeños o excesivamente grandes (Baca y Seminario, 2012).

Según datos de la OMS, más de 43 millones de personas entre 12 y 35 años padecen una pérdida auditiva discapacitante debido a diferentes causas (OMS, 2015). Pero no solo la pérdida de la capacidad auditiva es un efecto del ruido ambiental y ocupacional, sino que el riesgo de accidentes laborales, el estrés y problemas que afectan a las mujeres embarazadas incrementan con un aumento en el ruido (OSHA, 2005).

OMS en sus guías para el Ruido Urbano considera que los efectos del ruido y sus consecuencias de largo plazo sobre la salud se están generalizando. Por lo cual es esencial tomar acciones para limitar y controlar la exposición al ruido ambiental. Esas acciones deben estar respaldadas por una adecuada evaluación científica de los datos disponibles sobre los efectos del ruido, en particular, la relación dosis-respuesta. Esa relación constituye la base del proceso de evaluación y gestión de riesgos.

La contaminación acústica en una ciudad es catalogada por algunos organismos internacionales como el cuarto problema ambiental de mayor importancia a nivel de salud humana, proviene fundamentalmente de las actividades que desarrollan sus ciudadanos, y requiere que la administración responsable disponga de una política de actuación que garantice unos niveles de calidad de vida aceptables (MER, 2011). En datos aportados por la Organización de Salud y Medio Ambiente de Andalucía (OSMAN) en $80 \%$ del ruido en ambientes urbanos se debe al tráfico, $10 \%$ a la industria y el restante $10 \%$ a otras fuentes (OSMAN, 2018).

Se debe considerar que aun cuando se posee un grado de subjetividad en el tema, pues los niveles de ruido para una persona pueden ser desagradables, para otra no lo son, existen parámetros adecuados y necesarios para cuantificar y catalogar los niveles de ruido (Kurakula 2007). Por ejemplo, en Costa Rica, existe el Reglamento para el control de contaminación y ruido (en adelante Reglamento), cuyo decreto ejecutivo es el No. 39200-S, en el cual se definen los niveles de ruido permitidos según la clasificación de la zona de muestreo. Las zonas clasificadas en el reglamento son: zona residencial, zona comercial, zona mixta, zona industrial, zona 
Ligia Bermúdez-Hidalgo - José Castro-Solis - Karla Vetrani-Chavarría - Igor Zúñiga-Garita

Manfred Murrell-Blanco - Douglas Barraza - Manuel Méndez-Flores

Creación de mapas acústicos en los cantones de Heredia, Alajuela y Belén, como indicadores de la contaminación sónica.

agrícola y pecuaria y zona de tranquilidad. Los niveles de ruido permitidos, tanto para emisor como receptor se muestran en el siguiente cuadro.

Cuadro 1. Límites de niveles de sonido en decibeles (dB) de acuerdo con el Reglamento para el control de contaminación y ruido (Decreto No. 39200-S)

\begin{tabular}{|c|c|c|c|c|c|c|c|c|c|c|c|}
\hline & \multicolumn{10}{|c|}{ ZONA RECEPTORA } \\
\hline & & \multicolumn{2}{|c|}{ Residencia } & \multicolumn{2}{|c|}{ Comercio } & \multicolumn{2}{|c|}{$\begin{array}{c}\text { Industria } 0 \\
\text { agrícola/ } \\
\text { pecuaria }\end{array}$} & \multicolumn{2}{|c|}{ Tranquilidad } & \multicolumn{2}{|c|}{ Mixta } \\
\hline \multirow{5}{*}{ 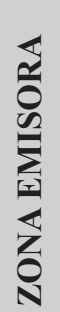 } & HORARIO & $\mathbf{D}^{<?>}$ & $\mathbf{N}^{<?>}$ & D & $\mathbf{N}$ & D & $\mathbf{N}$ & D & $\mathbf{N}$ & D & $\mathbf{N}$ \\
\hline & Residencial & 65 & 45 & 65 & 55 & 70 & 60 & 50 & 45 & 60 & 50 \\
\hline & Comercial & 65 & 45 & 65 & 55 & 75 & 65 & 50 & 45 & 60 & 50 \\
\hline & $\begin{array}{l}\text { Industria-agrícola- } \\
\text { pecuaria }\end{array}$ & 65 & 45 & 70 & 65 & 75 & 75 & 50 & 45 & 60 & 50 \\
\hline & Mixta & 60 & 50 & 60 & 50 & 60 & 50 & 50 & 45 & 60 & 50 \\
\hline
\end{tabular}

Fuente: Ministerio de Salud, 2015.

Existen varios factores reconocidos que contribuyen al aumento de la generación de ruido, en los que se encuentra una alta flota vehicular, que, al combinarse con un aumento en la densidad y altura de las construcciones existentes, así como concentración de personas y sonidos ambientales, lleva a crear espacios limitados, generando ambientes muy densos traduciéndose en zonas con niveles inaceptables de ruido ambiental (Kurakula 2007, Hong y Jeon 2014).

Este fenómeno puede ser abordado de varias formas, una de las cuales es la representación espacial a partir de mapas acústicos, los cuales pueden ser utilizados como herramientas diagnósticas con el fin de aproximar el comportamiento del ruido dentro de las ciudades, para lo cual se pueden combinar el análisis espacial a través de modelos matemáticos y algorítmicos, como elementos iniciales para ordenamiento territorial y construcción (Lazar et al 2007, Villatoro 2008, Murillo et al 2012). El desarrollo de los mapas para la representación del sonido trabaja bajo métodos de interpolación utilizando Sistemas de Información Geográfica (SIG), donde se pueden seleccionar distintos métodos según el comportamiento y arreglo espacial de los datos. 
Ligia Bermúdez-Hidalgo - José Castro-Solís - Karla Vetrani-Chavarría - Igor Zúñiga-Garita

Manfred Murrell-Blanco - Douglas Barraza - Manuel Méndez-Flores

Development of acoustic maps in Heredia, Alajuela and Belén counties, as indicators of noise pollution.

\section{Marco metodológico}

a. Área de estudio

La selección de los puntos de muestreo en los diferentes centros urbanos de los cantones de Alajuela y Belén se realizó mediante la definición de sitios de interés por las respectivas municipalidades, denuncias por ruido presentadas al Ministerio de Salud y otros puntos específicos.

Para la ubicación de los puntos de muestreo, se realizó la delimitación geográfica del área de estudio de cada uno de los cascos urbanos de los cantones. En el caso de Heredia, el establecimiento de los puntos de muestreo se realizó por parte del máster Marvin Alfaro, funcionario de la municipalidad, y fue a través de la distribución de los puntos en forma sistemática dejando una cuadra de por medio, de esta forma se definieron 83 puntos de muestreo, así como se muestra en la figura 1.

Figura 1. Ubicación geográfica de los sitios de muestreo, Heredia, Costa Rica, 2015.

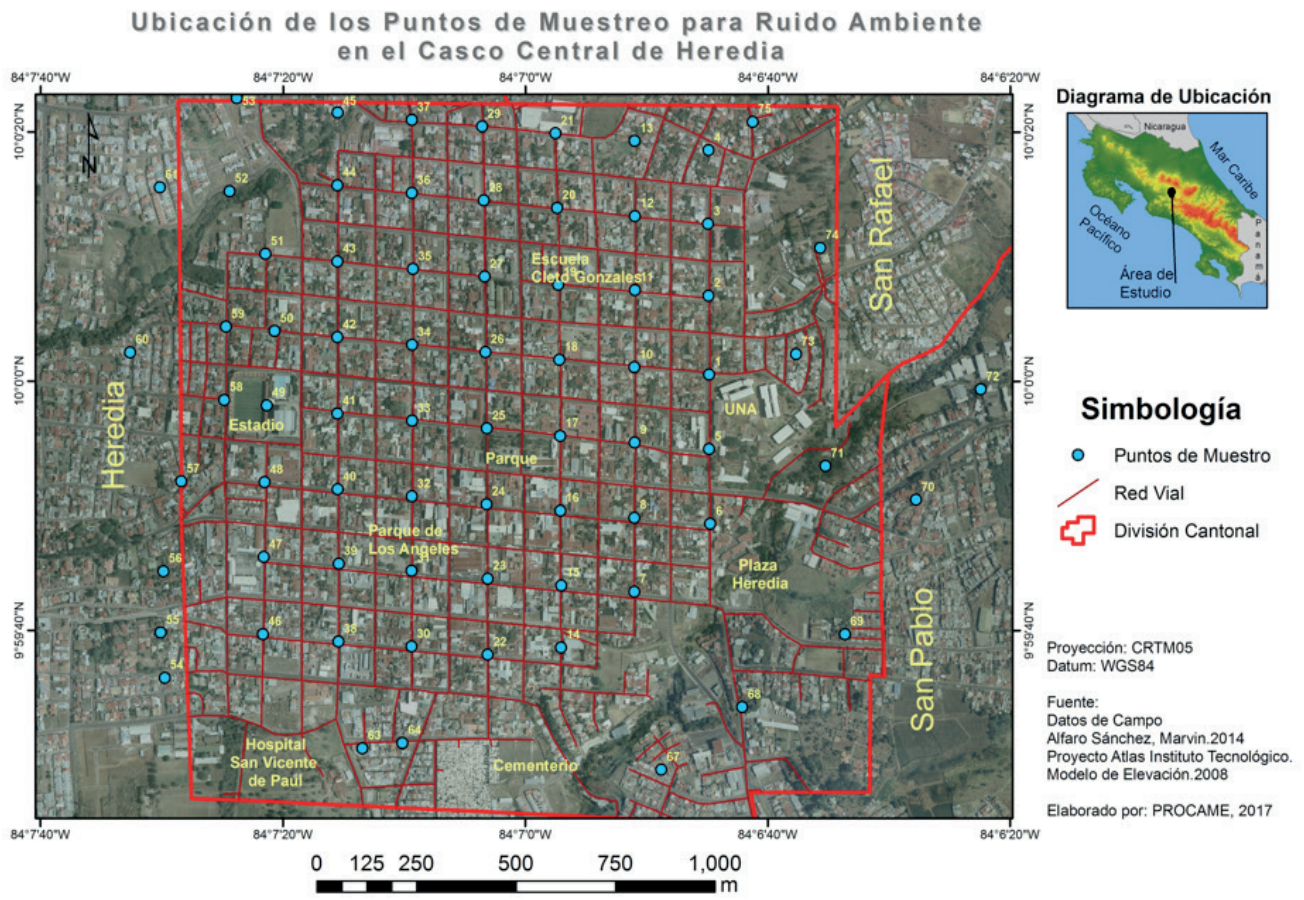


Ligia Bermúdez-Hidalgo - José Castro-Solis - Karla Vetrani-Chavarría - Igor Zúñiga-Garita

Manfred Murrell-Blanco - Douglas Barraza - Manuel Méndez-Flores

Creación de mapas acústicos en los cantones de Heredia, Alajuela y Belén, como indicadores de la contaminación sónica.

Con respecto a la definición de los sitios de muestreo de Alajuela, se establecieron a partir de la revisión de expedientes sobre denuncias por contaminación acústica, según información del Ministerio de Salud. Además, al ser este estudio un plan piloto, la municipalidad de dicho cantón incorporó otros sitios, en los cuales se obtendría información relevante para el ordenamiento territorial. En este sentido, se definieron 16 puntos de muestreo, los cuales se muestran en la figura 2.

Figura 2. Ubicación geográfica de los sitios de muestreo, Alajuela, Costa Rica, 2015.

Ubicación de los Puntos de Muestreo para Ruido Ambiente según denuncias, Alajuela
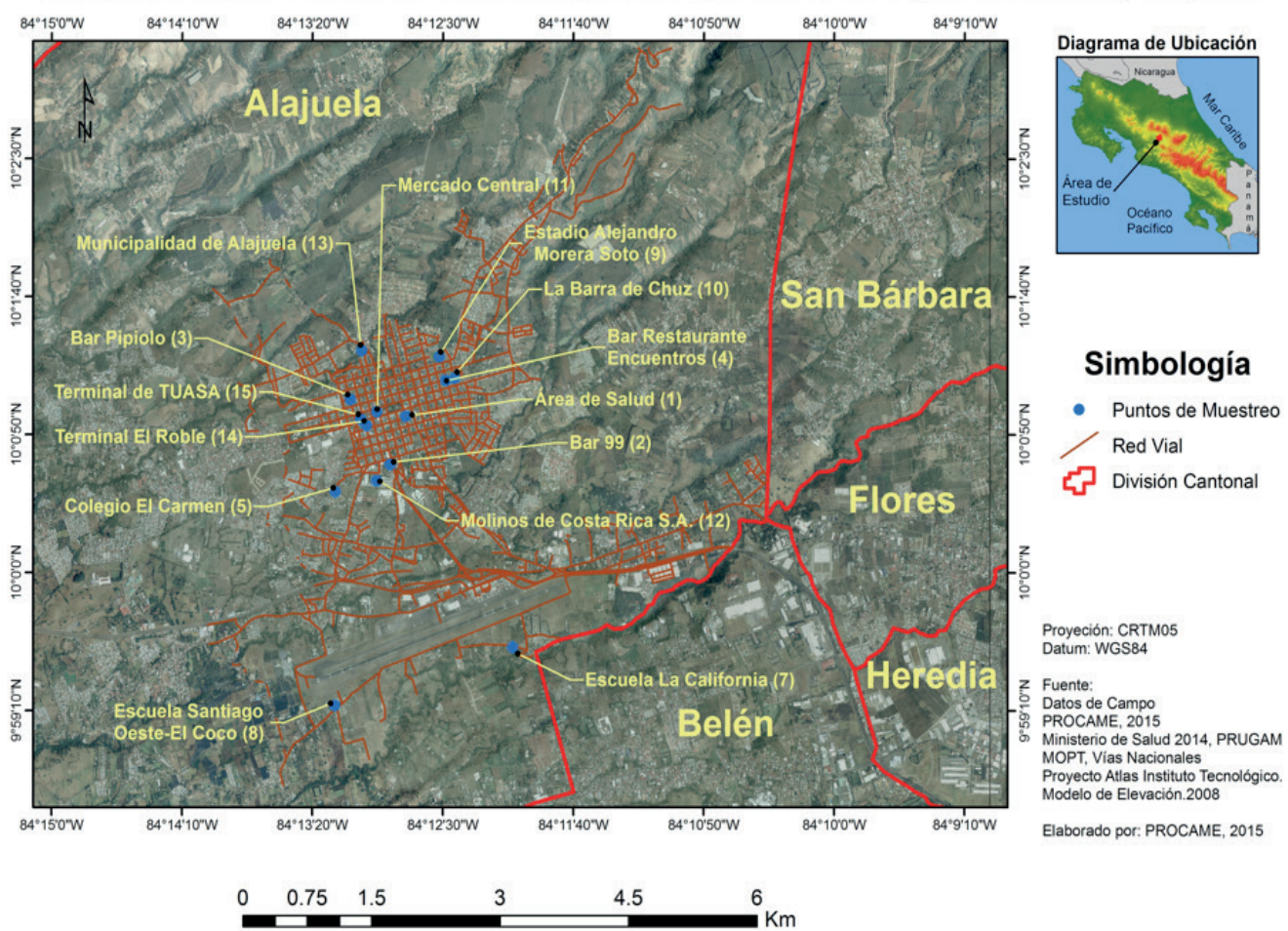

La definición de los puntos de muestreo en el casco urbano del cantón de Belén partió de una base de datos sobre denuncias realizadas específicamente en ciertos lugares, cuya información fue aportada por la municipalidad. Estos puntos fueron georreferenciados y mapeados, para 
Ligia Bermúdez-Hidalgo - José Castro-Solís - Karla Vetrani-Chavarría - Igor Zúñiga-Garita Manfred Murrell-Blanco - Douglas Barraza - Manuel Méndez-Flores

Development of acoustic maps in Heredia, Alajuela and Belén counties, as indicators of noise pollution.

proseguir con la toma de datos y la ubicación geográfica de éstos se pueden observar en la figura 3.

Figura 3. Ubicación geográfica de los sitios de muestreo, Belén, Costa Rica, 2015.

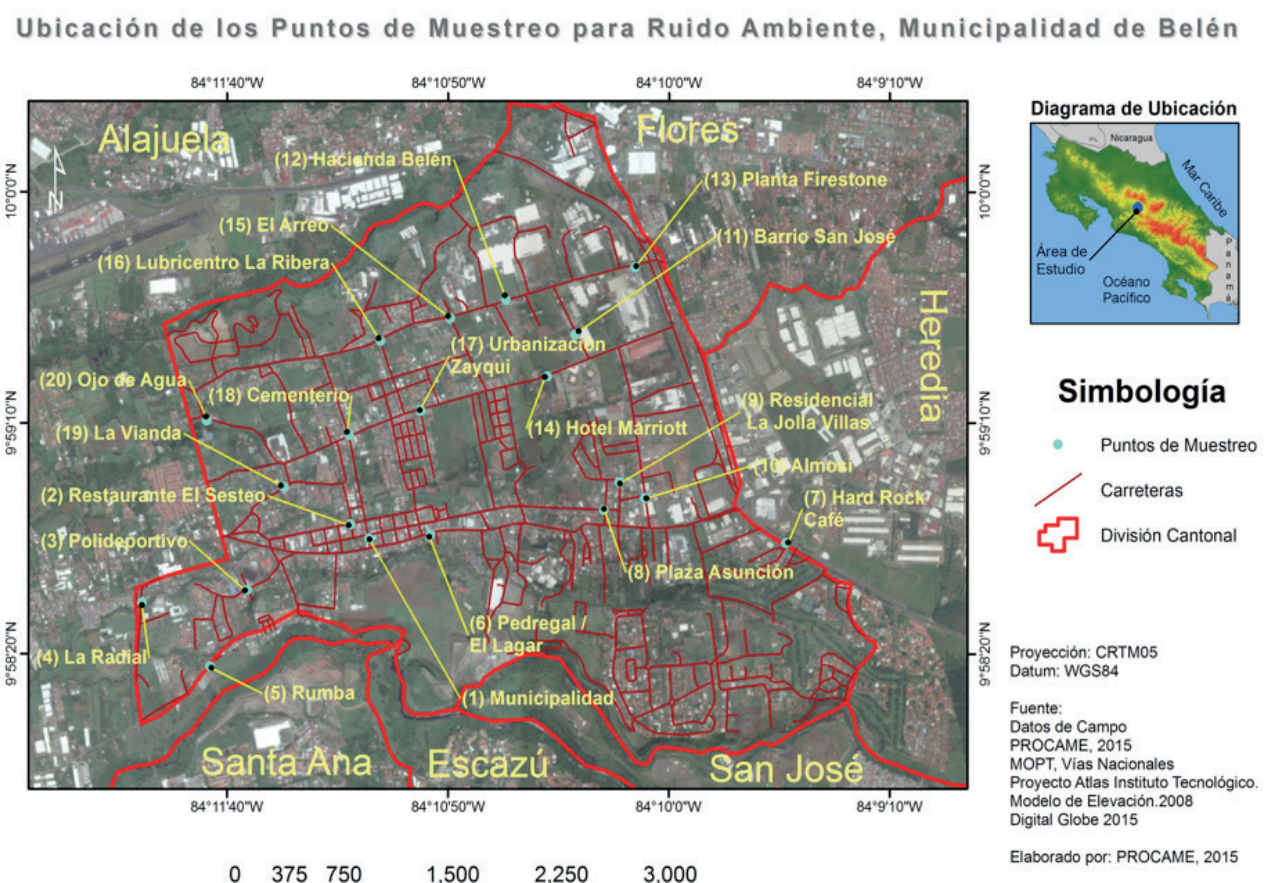

\section{b. Toma de datos y determinación del intervalo temporal de medición}

Para el casco urbano de Heredia, la toma de datos se realizó utilizando la aplicación para teléfonos móviles Metal Med db, con una duración de 5 min por ubicación. La toma de los datos se realizó en horario diurno en distintas horas del día a lo largo de aproximadamente tres meses de medición para cubrir todos los puntos. En Alajuela los datos se tomaron con la utilización de sonómetros Extech SN004 y QuestSoundPro, con una duración de 5 min con 15 repeticiones por ubicación. La toma se llevó a cabo en horario diurno, que según el Reglamento lo define de 6:00 a.m. a 8:00 p.m., durante 
Ligia Bermúdez-Hidalgo - José Castro-Solís - Karla Vetrani-Chavarría - Igor Zúñiga-Garita

Manfred Murrell-Blanco - Douglas Barraza - Manuel Méndez-Flores

Creación de mapas acústicos en los cantones de Heredia, Alajuela y Belén, como indicadores de la contaminación sónica.

cinco meses, de febrero a junio del año 2015. Para Belén, el método de toma de datos fue realizado con el sonómetro QuestSoundPro, con una duración de 5 min con 15 repeticiones por ubicación y se realizó para los dos horarios, diurno y nocturno, donde en el primero se consideraron las horas picos es de 6:00 a.m. a 9:00 a.m. y de 4:00 p.m. a 7:00 p.m., para un total de $6 \mathrm{~h}$, y en el segundo se tiene una distribución de 8:00 p.m. a 6:00 a.m. para un total de $10 \mathrm{~h}$. Esta toma se realizó en 3 meses, de octubre a diciembre del 2015.

\section{c. Medición de campo y procesamiento de información}

Para las mediciones de campo, se utilizaron instrumentos de medición clase 1 y 2, según lo establece la norma IEC 61672-1:2001, y los cuales fueron calibrados previamente y verificados posteriormente a cada serie de mediciones. En el momento de la toma de datos el equipo de medición se encontró a una altura aproximada de 1,5 $\mathrm{m}$ del nivel del suelo, con un ángulo de declinación de $30^{\circ}$ a $60^{\circ}$ y una distancia con respecto al cuerpo del operario de $0,5 \mathrm{~m}$.

Debido a que las mediciones se realizaron en el exterior, se utilizó una pantalla anti viento y a la vez se obtuvieron variables relacionadas como temperatura, presión atmosférica y velocidad del viento anterior y posterior a la toma de los datos. Es importante señalar, que no se tomaron datos mientras las condiciones meteorológicas fueran adversas, como, por ejemplo, fuertes vientos, tormentas eléctricas, u otros que afecten la medición o el equipo.

Una vez obtenida la información de las mediciones mensuales, se procedió a ingresar los datos al módulo de Excel "Mediciones y Cálculo de Incertidumbre Ambiental” (Microsoft 2013), el cual es parte del programa "Ruido y su control". Mediante el procesamiento de la información se calcularon los siguientes datos:

Nivel de presión sonora máximo, el cual determinó el valor del ruido más elevado obtenido durante el día de medición.

Nivel de presión sonora mínimo, que determinó el valor del ruido más bajo obtenido durante el día de medición. 
Sumatoria de ruido, donde se determinó la sumatoria logarítmica de los niveles de presión sonora del total de las mediciones durante el día de medición y que se expresa según la siguiente fórmula:

$$
\mathrm{L}_{\text {presultado }}=10 * \log \left(10^{\frac{\mathrm{L}_{\mathrm{p} 1}}{10}}+10^{\frac{\mathrm{L}_{\mathrm{pg}}}{10}}+10^{\frac{\mathrm{L}_{\mathrm{pg}}}{10}}+\cdots+10^{\frac{\mathrm{L}_{\mathrm{pn}}}{10}}\right)
$$

Nivel de presión sonora continuo equivalente, o nivel continuo equivalente (Leq), el cual contiene la misma energía que el ruido medido en una cantidad definida de tiempo. Un aspecto por considerar es que el ruido debe ser constante, por lo que se puede comparar el riesgo de daño auditivo ante la exposición a diferentes tipos de ruido, si se usa en la ponderación A se conoce como LAeq (Rejano, 2000). El cálculo del valor cuadrático medio de la presión sonora pondera a un período de observación se obtiene bajo esta expresión:

$$
\mathrm{L}_{\mathrm{Aeq}, \mathrm{T}}=10 * \log \left[\frac{1}{\mathrm{~T}} \int_{\mathrm{t}_{1}}^{\mathrm{t}_{\mathrm{2}}}\left(\frac{\mathrm{p}_{\mathrm{A}}^{2}(\mathrm{t})}{\mathrm{p}_{\text {ref }}^{2}}\right) \cdot \mathrm{dt}\right](\mathrm{dBA})
$$

Incertidumbre. Para cada una de las mediciones realizadas, con excepción de los datos de Heredia, se calculó la incertidumbre de los niveles de presión sonora, la cual depende de los siguientes factores: la fuente sonora y el intervalo de tiempo de medida, las condiciones de operación (repetibilidad), las condiciones del suelo y el clima, la distancia a la fuente, el método de medida y la instrumentación y del sonido residual. La incertidumbre de medida se expresa como una incertidumbre expandida basada en la incertidumbre típica combinada, multiplicada por un factor de cobertura de 2, que proporciona una probabilidad de cobertura de aproximadamente el $95 \%$ (el cálculo de la incertidumbre se basó en lo establecido por la Norma ISO 1996-2: 2009). En la figura 4 se presentan las fuentes. 
Ligia Bermúdez-Hidalgo - José Castro-Solis - Karla Vetrani-Chavarría - Igor Zúñiga-Garita

Manfred Murrell-Blanco - Douglas Barraza - Manuel Méndez-Flores

Creación de mapas acústicos en los cantones de Heredia, Alajuela y Belén, como indicadores de la contaminación sónica.

Figura 4. Componentes de incertidumbre en medición de ruido ambiente (ISO, 2009).

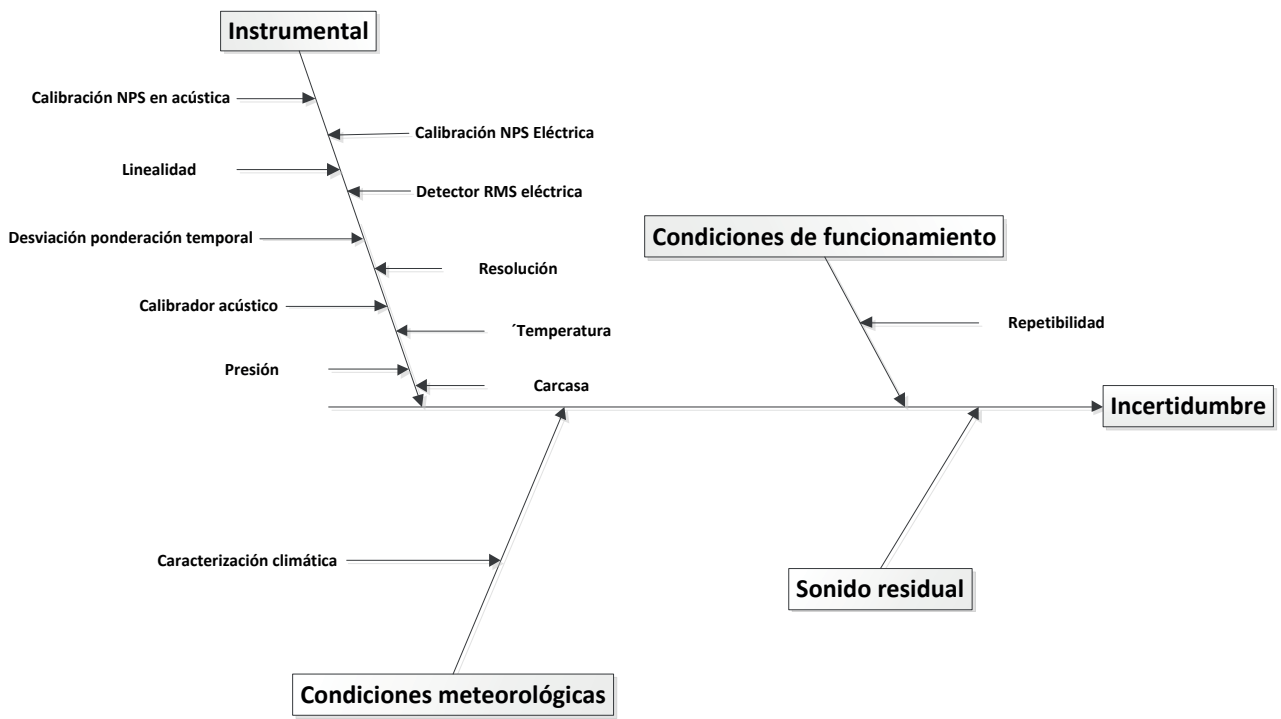

\section{d. Mapeo de la información}

Para ligar la información de campo con los puntos de muestreo, se creó una hoja de cálculo (Microsoft), donde se colocaron los valores medidos con un identificador único, el cual debía ser igual al archivo cartográfico vectorial con la ubicación de las coordenadas. Hecho lo anterior, se procedió a ligar las bases de datos por medio del programa especializado ArcGIS v. 10.3 (ESRI 2014) a través de una unión virtual de tablas de atributos, utilizando el identificador único como enlace entre los archivos y, posteriormente, se guardó como un nuevo tema. Este proceso se realizó para cada una de las visitas de campo, generando una base de datos mensual de las mediciones realizadas.

La creación de la máscara de análisis se generó de la combinación de las capas temáticas de distritos de Costa Rica (Geotecnologías a partir de hojas 1:50000 SF) y red de caminos (IGN a partir de hojas 1:50000, 2004), para este último se creó un área de amortiguamiento (buffer) de aproximadamente $10 \mathrm{~m}$, con el fin de unir los dos temas, utilizando mapas de las municipalidades, se extrajo la información catastral de predios con un sistema de georeferenciación y reclasificación de los valores para su posterior verificación. Toda la información generada está proyectada en 
Costa Rica Transversal de Mercator (CRTM05) con el elipsoide de referencia WGS84.

El análisis espacio-temporal mensual para los sets de datos se realizó a partir de un análisis variográfico para determinar el comportamiento de las mediciones (Yepes et al 2009) utilizando el módulo de geoestadística para ArcGIS (ESRI 2014). Así mismo, se generaron varios modelos en formato raster, considerando la cantidad de información disponible y obtener el más representativo según el comportamiento esperado.

Utilizando el módulo de interpolación, herramientas de análisis espacial (ArcGIS 2014), se trabajó con interpoladores IDW (Correa et al 2008, Villatoro et al 2008, Yepes et al 2009, Caracausi 2014), dado que representó de mejor forma la distribución espacial de los datos. El método asigna valores de más peso a los puntos más cercanos a los puntos de medición, utilizando un algoritmo sobre distancias inversas, asumiendo una estructura lineal de los datos.

A raíz de esto, se creó un modelo mensual para las mediciones, utilizando una resolución espacial de 2 m x 2 m y se utilizó algebra de mapas para realizar la combinación de los modelos interpolados mensuales, tanto diurnos como nocturnos (uno para la sumatoria de ruido y otro nivel continuo equivalente), donde el resultado final fueron las representaciones medias del comportamiento del ruido para todo el período de medición.

\section{Resultados}

\section{a. Niveles de ruido en Heredia}

De acuerdo con el Reglamento, el casco central de Heredia está conformado de tres zonas, residencial, comercial y tranquilidad. En cuanto a la zona residencial (la de mayor superficie), se determinó que el 8,6\% de los datos obtenidos de niveles de ruido presenta valores menores o iguales a $65 \mathrm{~dB}(\mathrm{~A})$, mientras que el 91,4\% restante supera este valor, con datos que llegan hasta $104 \mathrm{~dB}(\mathrm{~A})$. La zona comercial, ubicada principalmente en el centro del casco urbano de Heredia, mostró el mismo comportamiento que el área residencial, donde $57 \%$ de los sitios muestreados presentan niveles de ruido entre $73 \mathrm{~dB}(\mathrm{~A})$ y $85 \mathrm{~dB}(\mathrm{~A})$, mientras que el $43 \%$ restante presenta valores mayores a $85 \mathrm{~dB}(\mathrm{~A})$. En la zona de tranquilidad, ubicada al sur del casco urbano (en los alrededores del Hospital), se mostraron niveles de ruido mayores a $50 \mathrm{~dB}(\mathrm{~A})$, cuyo valor es el límite permitido como sitio receptor 
Ligia Bermúdez-Hidalgo - José Castro-Solis - Karla Vetrani-Chavarría - Igor Zúñiga-Garita

Manfred Murrell-Blanco - Douglas Barraza - Manuel Méndez-Flores

Creación de mapas acústicos en los cantones de Heredia, Alajuela y Belén, como indicadores de la contaminación sónica.

según el Reglamento. Si se observa el mapa acústico de Heredia (Figura 5), las áreas de color verde, ubicadas en su mayoría en la periferia, son las que presentan los niveles de ruido más bajos, no obstante, las zonas con niveles de ruido inferiores a $65 \mathrm{~dB}(\mathrm{~A})$ no superan el $10 \%$ de los sitios de muestreo (zonas de color verde oscuro). Esto quiere decir que aproximadamente el $90 \%$ de la superficie del casco central de Heredia tienen valores de ruido superiores $65 \mathrm{~dB}(\mathrm{~A})$. Las áreas que muestran los mayores niveles de ruido se ubican en el sector de la Universidad Nacional y en la salida de Heredia hacia San Joaquín de Flores y Alajuela, con valores establecidos en un intervalo de 94,6 dB(A) a $104 \mathrm{~dB}(\mathrm{~A})$, y los sitios donde se presentan los valores de ruido más bajos, corresponden a los sectores residenciales de la periferia, principalmente al norte y sur, como, por ejemplo, Monte Bello y Corazón de Jesús en el Norte y al sur del Cementerio y Guayabal.

Figura 5. Mapa acústico en los sitios de muestreo para el casco urbano del cantón Central de Heredia, Costa Rica

Comportamiento del Ruido Ambiente en el Casco Central de Heredia
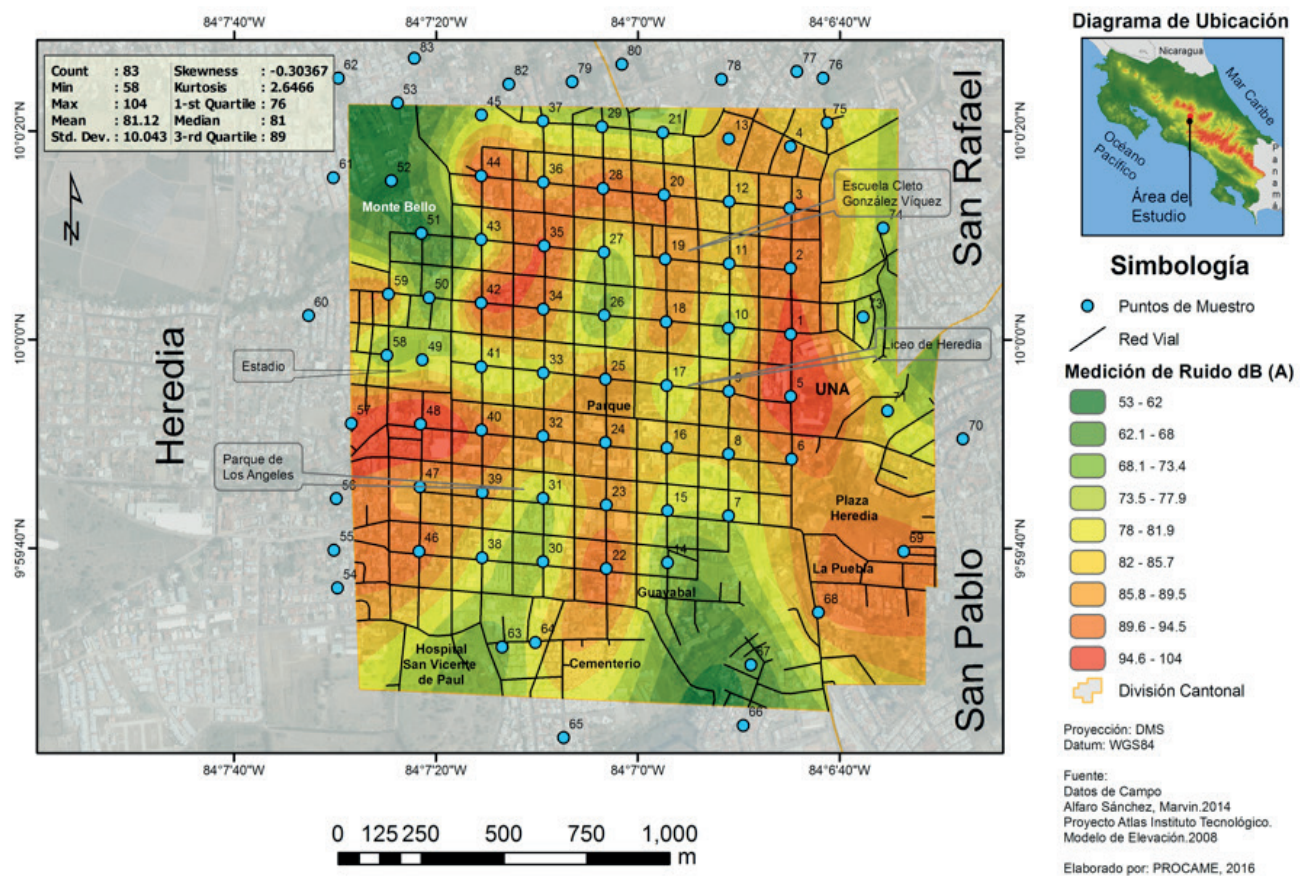


\section{b. Niveles de ruido en Belén}

Como se citó en la metodología, el muestreo del estudio en el cantón de Belén se realizó durante tres meses y en dos horarios, diurno y nocturno. Para efectos de este artículo, y con el afán de realizar un análisis comparativo, se muestran solamente los datos obtenidos del mes de octubre para el año 2015 en los dos horarios, los puntos de muestreo fueron ubicados en diversas zonas según la categoría establecida por el Reglamento, siempre predominando la zona residencial. En cuanto a los resultados obtenidos, para el horario diurno se obtuvieron niveles de ruido que oscilan entre 41,6 dB(A) (sitio Rumba) y 74,8 dB(A) (sitios el Lagar y el Arreo). Cabe señalar, que el sitio el Arreo se ubica en zona industrial y el sitio Lagar se localiza en zona residencial a pesar de ser una actividad comercialindustrial. En relación con el sitio Rumba, cuya actividad se cataloga como comercial, está ubicado en zona residencial. En la figura 6, se observa que en el casco urbano del cantón de Belén, los sectores sureste y suroeste presentaron los niveles de ruido más bajos (en el mapa se muestran de color verde), que coinciden con zonas residenciales y de menor tránsito vehicular. Por otro lado, los sitios de muestreo con mayores niveles de ruido se ubican en el centro y norte del casco urbano, específicamente en las áreas donde se localizan industrias y comercios y a la vez, hay mayor afluencia de tránsito vehicular y de maquinaria pesada. 
Ligia Bermúdez-Hidalgo - José Castro-Solis - Karla Vetrani-Chavarría - Igor Zúñiga-Garita

Manfred Murrell-Blanco - Douglas Barraza - Manuel Méndez-Flores

Creación de mapas acústicos en los cantones de Heredia, Alajuela y Belén, como indicadores de la contaminación sónica.

Figura 6. Mapa acústico en los sitios de muestreo en horario diurno para el casco urbano del cantón Belén, Heredia, Costa Rica.

Nivel Continuo Equivalente Diurno para Ruido Ambiente, Cantón de Belén

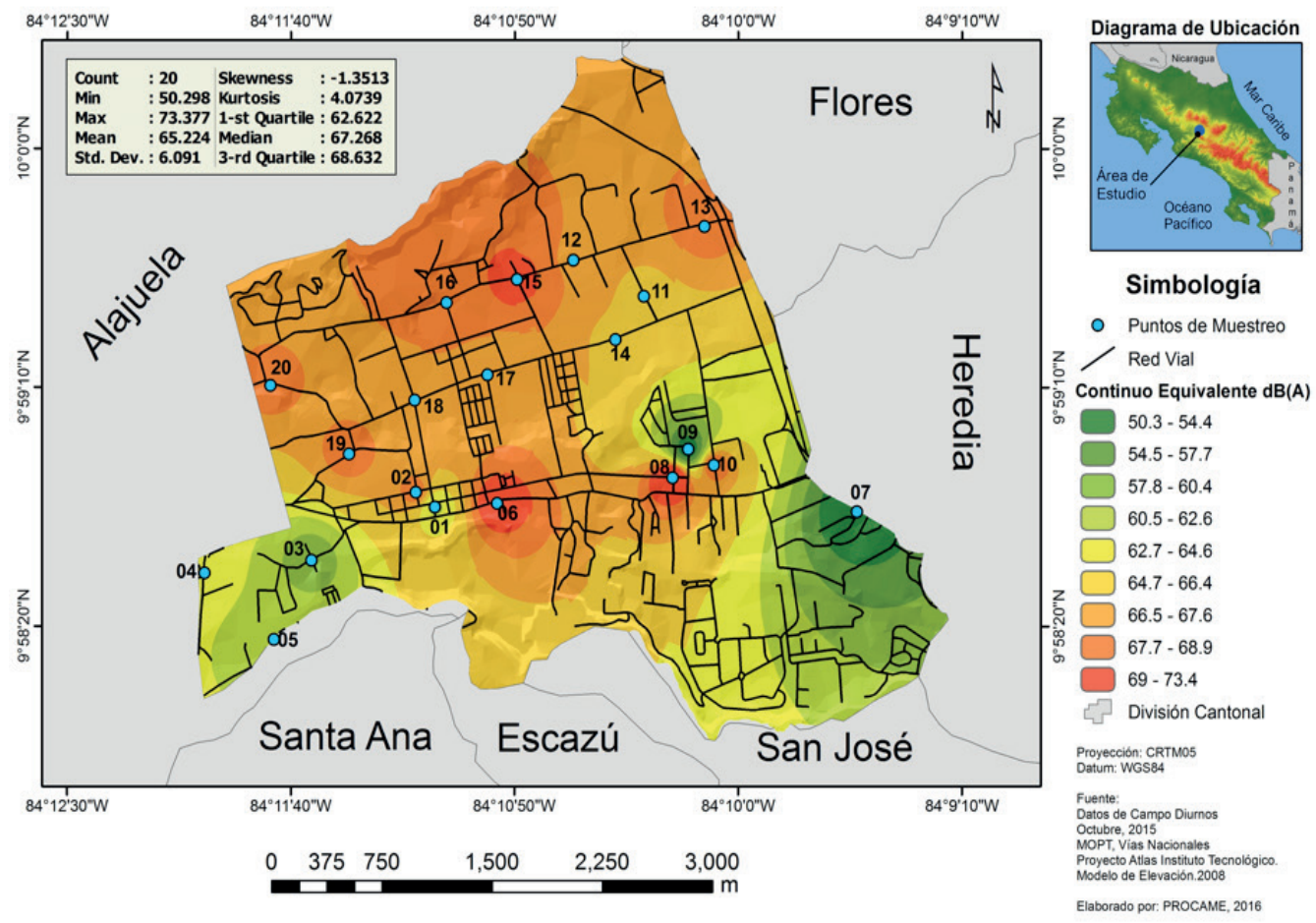

Si se hace una comparación de los niveles de ruido entre el horario diurno y el nocturno, se pueden observar diferencias significativas, debido a que en el segundo se da la presencia de mayores áreas de color verde y los colores rojos que muestran los valores más altos de ruido empiezan a disminuir. Sin embargo, es interesante observar el sector del punto 07, correspondiente a Hard Rock Café, en el cual hay un incremento en los niveles de ruido en el horario nocturno, aspecto que se justifica por el tipo de actividad que se realiza en este sitio (centro nocturno), aun así no supera los $60 \mathrm{~dB}(\mathrm{~A})$ (misma situación sucede en el sitio Rumba que pasa de $41,6 \mathrm{~dB}(\mathrm{~A})$ en horario diurno a 55,3 $\mathrm{dB}(\mathrm{A})$ en horario nocturno), como se observa en la figura 7 
Figura 7. Mapa acústico en los sitios de muestreo en horario nocturno para el casco urbano del cantón Belén, Heredia, Costa Rica.

Nivel Continuo Equivalente Nocturno para Ruido Ambiente, Cantón de Belén

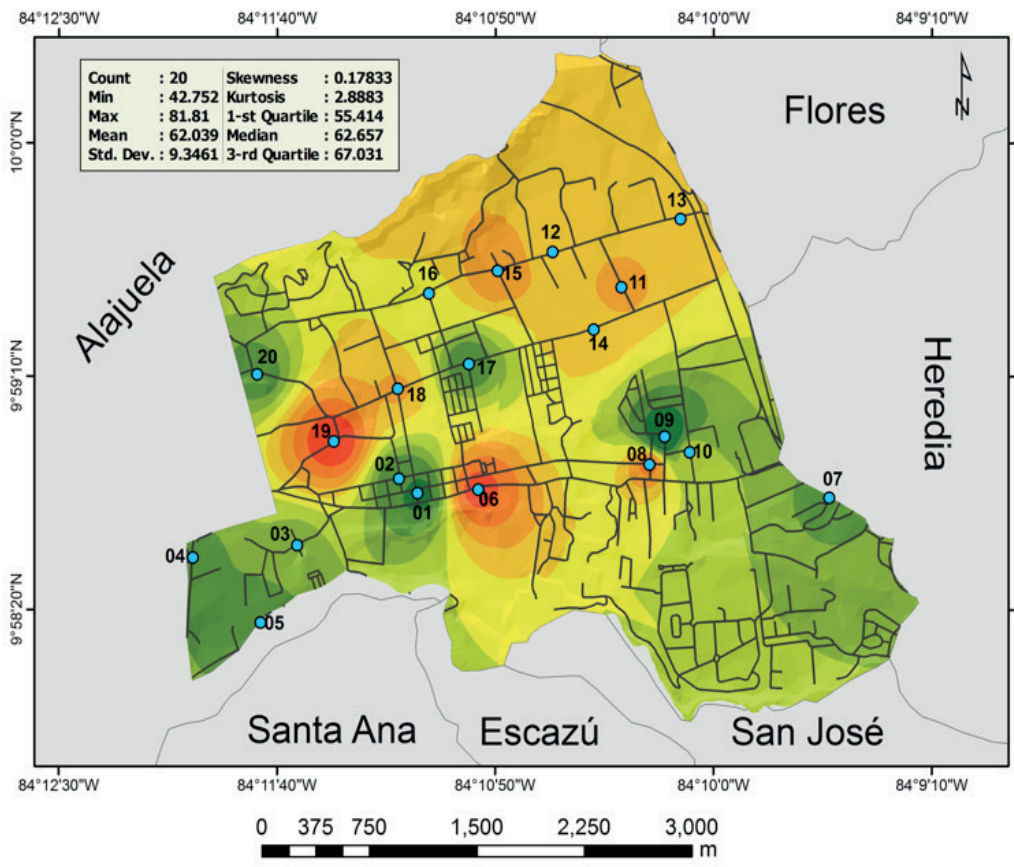

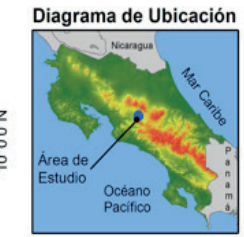

Simbología

- Puntos de Muestreo

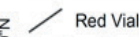

Continuo Equivalente dB(A)

$42.8-53.5$

$53.6-57.8$

$57.9-59.8$

$59.9-61.7$

$61.8-64$

$64.1-66.2$

$66.3-69.4$

$69.5 \cdot 74.2$

$74.3-81.7$

7 División Cantonal

Proyección: CRTMOS

Datum: WGS84

Fuente:

Datos de Campo Nocturnos Octubre, 2015 Proyecto Attas Instituto Tecnológion. Modelo de Elevación.2008

Elaborado por. PROCAME, 2016

\section{c. Niveles de ruido en Alajuela}

Para efectos de los resultados de los niveles de ruido en el casco urbano del cantón de Alajuela, se hace un análisis comparativo de los cinco meses en los que se tomaron los datos. Las áreas muestreadas se ubican en tres diferentes zonas: Zona urbano-residencial, zona comercial y zona industrial. De acuerdo con la información obtenida de este estudio, se determinó que $67.2 \%$ de la zona residencial, correspondió a sitios que registran un nivel continuo equivalente de ruido menor o igual a $65 \mathrm{~dB}(\mathrm{~A})$ y el restante $32.8 \%$ son áreas que sobrepasan este valor. Además, se obtuvo dentro de los principales resultados, que la zona con los niveles de ruido más altos corresponde a la Comercial (ZC). En este sentido, en la figura 8, se puede observar en promedio cuales son los niveles de ruido de acuerdo 
Ligia Bermúdez-Hidalgo - José Castro-Solis - Karla Vetrani-Chavarría - Igor Zúñiga-Garita

Manfred Murrell-Blanco - Douglas Barraza - Manuel Méndez-Flores

Creación de mapas acústicos en los cantones de Heredia, Alajuela y Belén, como indicadores de la contaminación sónica.

con los distintos sectores, donde los valores más altos se ubican en los sitios del centro del distrito de Alajuela y conforme se alejan de éste, los niveles de ruido tienden a disminuir, principalmente en el sector sur. Los sitios ubicados en los alrededores del Estadio Morera Soto (zona comercial) presentan valores de ruido menores a $65 \mathrm{~dB}(\mathrm{~A})$, al igual que los sitios de las escuelas La California y Santiago Oeste-El Coco y el colegio El Carmen (zonas urbano-residenciales). Los sitios con niveles de ruido más altos presentan valores mayores a $65 \mathrm{~dB}(\mathrm{~A})$, superando en promedio los $70 \mathrm{~dB}(\mathrm{~A})$ y se ubican en las Zonas Comerciales, cuatro de estos sitios son: Bar Pipiolo, Bar 99, Terminal el Roble y TUASA.

Figura 8. Mapa acústico en los sitios de muestreo en horario para el casco urbano del cantón Alajuela, Costa Rica.

Nivel Continuo Equivalente dentro de Casco Central de Alajuela

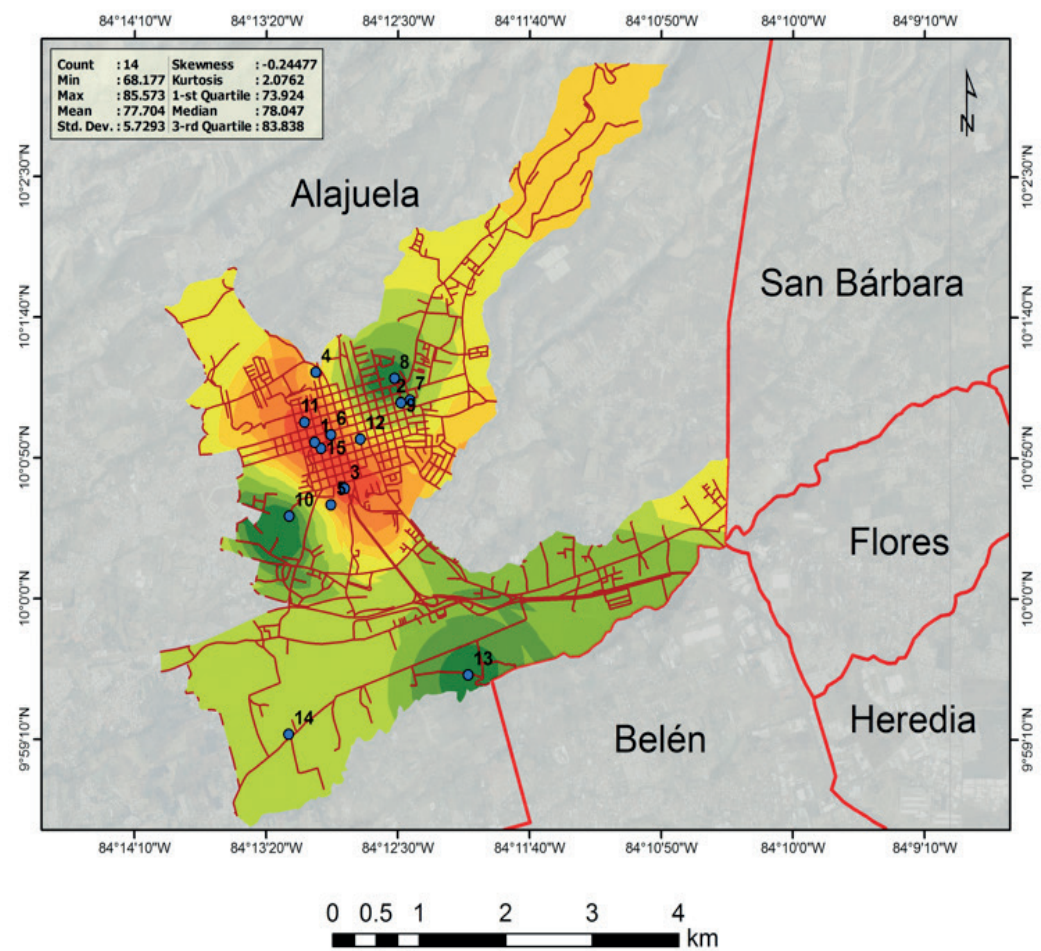

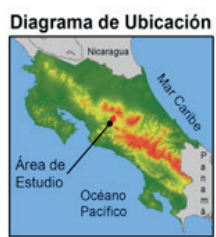

Simbología

- Puntos de Muestreo

Red Vial

Continuo Equivalente Medio dB(A)

$60.83-62.27$

$62.28-63.16$

$63.17-64.18$

$64.19-65.36$

$65.37-66.29$

$66.3-67.14$

$67.15-68.12$

$68.13-69.34$

$69.35-71.63$

División Cantonal

Proyeción: CRTM05

Datum: WGS84

Fuente:

Datos de Campo

PROCAME, 2015 MOPT, Vias Nacionales Proyecto Atlas Instituto Tecnológico. Modelo de Elevación. 2008

Elaborado por: PROCAME, 2015 


\section{Discusión de resultados}

Bruel y Kjaer (2002), citado por Kurakula (2007), hacen mención que el ser humano posee umbrales de intensidad de la presión del sonido, donde se obtiene un intervalo a partir de $0 \mathrm{~dB}(\mathrm{~A})$ hasta $130 \mathrm{~dB}(\mathrm{~A})$, en este último se encuentra el umbral máximo que produce dolor. A partir de 50 $\mathrm{dB}(\mathrm{A})$ los niveles de ruido producen problemas para poder comunicarse, $\mathrm{y}$ valores cercanos a $90 \mathrm{~dB}(\mathrm{~A})$ se consideran como peligrosos para el oído, principalmente si la exposición es continua.

Con respecto a los resultados obtenidos de las tres áreas de estudio (Heredia, Belén y Alajuela), se pudieron identificar niveles de ruido con valores mayores a $50 \mathrm{~dB}(\mathrm{~A})$, que como se citó en el párrafo anterior, ya repercute en la comunicación. Ahora, si se analizan los valores de niveles de ruido con respecto a los límites establecidos por el Reglamento para el control de contaminación y ruido en Costa Rica, muchas de las zonas de estudio se ubican sobre los límites permitidos, tanto en horario diurno como nocturno. Por ejemplo, en Heredia, el $90 \%$ de los datos obtenidos superan los $65 \mathrm{~dB}(\mathrm{~A})$, cuyo valor corresponde al límite permitido en las zonas residenciales y comerciales en el horario diurno. Por otro lado, en Belén, de los 20 sitios muestreados, en horario diurno se detectaron 6 que sobrepasan los límites permitidos por el Reglamento, mientras que en el horario nocturno hay 17 sitios que sobrepasan los límites, cuya diferencia se justifica con la presencia de un aumento de los niveles de ruido del horario diurno al nocturno en 13 de los sitios. Esto lleva a pensar que hay una falta de control por las instancias correspondientes en la noche del tema ruido para algunas áreas donde hay, principalmente, comercios e industrias.

Por último, en Alajuela, si se obtiene un promedio de los 5 meses analizados y con respecto a cada una de las categorías de uso, la zona residencial presenta en el horario diurno 3 sitios de 5 con niveles de ruido que sobrepasan los límites permitidos por el Reglamento, en la zona comercial 6 sitios de un total de 9 con la misma situación y en la zona industrial los dos sitios muestreados tienen niveles de ruido por debajo de los límites permitidos. Esto quiere decir que de 16 áreas muestreadas en Alajuela 9 puntos no cumplen con los parámetros establecidos en el reglamento.

Partiendo de la dinámica de la contaminación sónica en las áreas urbanas, como se pudo observar en el estudio de estos tres cantones, es 
Ligia Bermúdez-Hidalgo - José Castro-Solís - Karla Vetrani-Chavarría - Igor Zúñiga-Garita

Manfred Murrell-Blanco - Douglas Barraza - Manuel Méndez-Flores

Creación de mapas acústicos en los cantones de Heredia, Alajuela y Belén, como indicadores de la contaminación sónica.

importante considerar su diseño o rediseño utilizando herramientas como los mapas acústicos, que permiten identificar los sitios de mayor o menor nivel de ruido en los distintos horarios.

$\mathrm{Al}$ tener áreas pobladas de alta densidad con accesos por carretera, afecta la calidad de vida en las zonas residenciales, resultando en algunos casos en la reducción del valor económico de las propiedades, lo que implica que se deben de buscar materiales especiales que ayuden a aislar el sonido excesivo. Así mismo, ese fenómeno afecta no solo la salud humana, sino que también otros aspectos como la productividad, efectos psicológicos, pues las personas pueden experimentar problemas de concentración, de comunicación o fatiga por la falta de sueño o de un ambiente adecuado para su desarrollo integral. (Kurakula 2007)

Kurakula (2007) considera varios factores que contribuyen a aumentar de los niveles de ruido en las zonas urbanas, uno es el aumento de la población, lo que contribuye a un alto volumen de tráfico combinado con un incremento de la intensidad de actividades comerciales, así como el establecimiento de oficinas gubernamentales y de ocio, lo que genera la creación de nueva infraestructura, que produce la creación de nuevos espacios para edificios y la reducción de áreas abiertas. Considerando los resultados obtenidos, se puede considerar que la naturaleza de la contaminación sónica dentro las carreteras y de las ciudades se genera por factores relacionados desde el sonido que genera los motores hasta la fricción generada entre el vehículo con superficie de rodaje y el aire. Mucha de la contaminación sónica depende del volumen de tránsito, velocidad de los automotores, proporción de vehículos pesados en la vía, así como los materiales con los cuales está construidas las calles.

Los mapas acústicos, en este sentido, representan una herramienta útil para la interpretación regional de los niveles de ruido, permitiendo una planificación urbana basada en la mejora de la calidad de vida al disminuir o minimizar la contaminación sónica mediante la implementación de medidas de confinamiento y de control vial, siendo el tránsito vehicular una de las principales fuentes del aumento del ruido.

\section{Conclusiones}

Se encontró que los cantones de Heredia, Belén y Alajuela, presentan niveles de presión sonora mayores a los permitidos en la legislación 
tanto para horario diurno como nocturno, notándose un aumento en el incumplimiento en horario nocturno.

Debido al más del $60 \%$ de los sitios muestreados sobrepasaron los límites establecidos por el Reglamento para el control de contaminación y ruido (decreto ejecutivo es el No. 39 200-S), se declara estas zonas con problemas de contaminación sónica.

En este caso, los datos fueron tomados de los tres cascos urbanos bajo estudio de los cantones de Heredia, Alajuela y Belén, cuentan con una cantidad significativa de población, de tránsito vehicular y de desarrollo comercial, por lo cual esto puede estar contribuyendo a que los niveles en estos sitios sean mayores a los permitidos.

Es importante que se utilicen herramientas como los mapas acústicos en la planificación urbana del país, ya que los niveles de ruido pueden ser relacionados con el crecimiento poblacional, desarrollo de actividades económicas y de flujo vehicular. Esto debe ser un factor considerar para la zonificación de los sitios de manera que interfieran lo menos posible con la calidad de vida de las personas.

\section{Referencias}

Geotecnologías (2014). Distritos de Costa Rica [capa vectorial] del Atlas Digital de Costa Rica 2014.

Caracausi, R. (2014). Application of geographic information systems (GIS) and noise mapping of noise in urban structures. The 21 International Congress on Sound and Vibration. China. 8pp.

Gray, A. (2017). These are the cities with the worst noise pollution. Recuperado de: https:/www.weforum.org/agenda/2017/03/ these-are-the-cities-with-the-worst-noise-pollution/

Presidencia de la República y Ministerio de Salud (2015). Decreto N 39200-S. 2015. Reglamento para el Control de Contaminación por Ruido. Diario Oficial La Gaceta 197. San José.

Hong, J. y Jeon, J. (2014). Soundscape mapping in urban contexts using GIS techniques. Hanyang University, Korea. Inter noise 2014, Melbourne, Australia, 16 - 19 November. 5 pp.

Instituto Geográfico Nacional (IGN). (2014). Redcamino 2014 [capa vectorial]. Escala 1: 50000. Hojas Cartográficas escala 1: 50000. 2004. Atlas Digital de Costa Rica 2014. 
Ligia Bermúdez-Hidalgo - José Castro-Solís - Karla Vetrani-Chavarría - Igor Zúñiga-Garita

Manfred Murrell-Blanco - Douglas Barraza - Manuel Méndez-Flores

Creación de mapas acústicos en los cantones de Heredia, Alajuela y Belén, como indicadores de la contaminación sónica.

Norma ISO 1996-2 (2009). Descripción, medición y evaluación del ruido ambiental, Parte 2: Determinación de los niveles de ruido ambiental. ES.

Kovak, C., Copeland, T., Elder, N., Thomas, N. y Ule, H. (2009). Acoustic Impact of the Green Corridor Action Group's Urban Design Using Acoustic Mapping. Canadian Acoustics 37 (4): 3-11.

Kurakula, V. (2007). A GIS-based approach for 3D noise modelling using $3 D$ city models. ITC. The Netherlands.

Lazar, J., Bruce, D., Giordiano, S., Levin, D., Little, C. ySlacum, W. (2007). Acoustic mapping and GIS aiden oyster restoration. Proceeding of Costal Zone 07. Portland, Oregon.

MER. (2011). Mapa Estratégico de Ruido de Madrid. Madrid. España.

Murillo, D., Ortega, I., Carrillo, J., Pardo, A., Rendón, J. (2012). Comparación de métodos de interpolación para la generación de mapas de ruido en entornos urbanos. Ing. USB med 1 (3): 62-68.

Rejano, M (2000). Ruido Industrial y Urbano. ES, Paraninfo Thomson Leaning..

Szczodrak, M., Kotus, J., Czyzewski, A., Kostek, B. (2013). The application of noise mapping tool deployed in grid infrastructure for creating noise maps of urban areas. Computer Science 14 (2) 231-242.

Villatoro, M., Henríquez, C., Sancho, F. (2008). Comparación de los interpoladores IDW y Kriging en la variación espacial de $\mathrm{pH}, \mathrm{CA}$, Cice y P del Suelo. Agronomía Costarricense 32 (1) 95-105.

Wang, B. y Kang, J. (2011). Effects of urban morphology on the traffic noise distribution through noise mapping: A comparative study between UK and China. Applied Acoustics 72, 556-568.

Yepes, D., Gómez, M., Sánchez, L. y Jaramillo, A. (2009). Metodología de elaboración de mapas acústicos como herramienta de gestión del ruido urbano caso Medellín. Dyna. No 158, 29-40.

Verdú, V. (2010). Medición y evaluación de la incertidumbre de auditorías en el aislamiento acústico. Universidad Politécnica de Valencia. España. 